\section{Participación de familiares cuidadores para el confort de \\ personas mayores hospitalizadas}

\section{Participation of family caregivers for the comfort of hospitalized elderly people

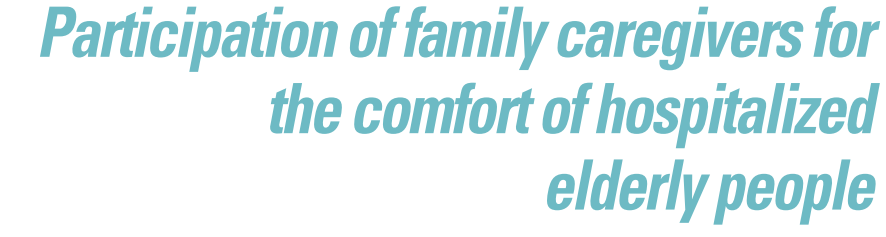

\section{RESUMEN}

Objetivo: Describir la participación del familiar cuidador para el confort de las personas mayores de 65 años hospitalizadas, según la percepción de las enfermeras y los familiares cuidadores en un hospital público de Lambayeque, Perú. Métodos: Investigación cualitativa exploratoria descriptiva; la muestra fue de 20 informantes previo consentimiento informado. Los datos fueron recolectados mediante la entrevista semiestructurada y procesados según análisis de contenido temático. Resultados: Emergieron tres categorías: a) participación en los cuidados básicos: confort del cuerpo; $b$ ) estrategias para el descanso

y sueño: confort del ambiente; $c$ ) afecto, ánimo y fe: contribución

al confort emocional y espiritual. Conclusiones: La permanencia hospitalaria genera incomodidades en las personas adultas mayores, pero el familiar cuidador participa activamente en la satisfacción de las necesidades básicas como la higiene, alimentación, tendido de cama, evitación del dolor y de los ruidos, iluminación adecuada, seguridad,

afecto, esperanza y fe; cuidados orientados a proporcionarles un entorno tranquilo y agradable que les ayuda en el descanso y sueńo, la adaptación, la recuperación y la calidad de vida. Es indispensable que el personal de enfermería capacite al familiar cuidador y lo prepare para colaborar en el cuidado tanto en la hospitalización como en el hogar.

PALABRAS CLAVE: Confort, hospitalización, familiar cuidador, persona mayor, enfermería.
Shirley Arminda Rodríguez Medina ${ }^{1}$

Rosa Jeuna Díaz Manchay ${ }^{2, *}$

Mirtha Flor Cervera Vallejos ${ }^{3}$

Antonieta de Jesús Banda Pérez ${ }^{4}$

Lisseth Dolores Rodríguez Cruz $^{5}$

Sonia Tejada Muñoz ${ }^{6}$

1. Licenciada en Enfermería. Escuela de Enfermería de la Universidad Católica Santo Toribio de Mogrovejo. Chiclayo. Perú. https://orcid.org/0000-0002-0368-7210

2. Doctora en Enfermería. Coordinadora de asuntos académicos de la Escuela de Enfermería de la Universidad Católica Santo Toribio de Mogrovejo. Chiclayo. Perú. https://orcid.org/0000-0002-2333-7963

3. Doctora en Enfermería. Directora de Escuela de Postgrado de la Universidad Católica Santo Toribio de Mogrovejo. Chiclayo, Perú. https://orcid.org/0000-0002-4972-1787

4. Estudiante de Doctorado en Ciencias de Enfermería, Campus Celaya Salvatierra Universidad de Guanajuato. Docente de la Facultad de Enfermería y Nutrición de la Universidad Autónoma de San Luis Potosí, México. https://orcid.org/0000-0001-95860641

5. Doctoranda en Ciencias de Enfermería de la Universidad de Navarra-España. Docente de la Escuela de Enfermería de la Universidad Católica Santo Toribio de Mogrovejo. Chiclayo. Perú. https://orcid.org/0000-0003-1742-9498

6. Doctora en Enfermería. Docente de la Universidad Nacional Toribio Rodríguez de Mendoza. Chachapoyas. Perú. https://orcid.org/0000-0002-1181-8540

*Autor para correspondencia.

Correo electrónico: rdiaz@usat.edu.pe (Rosa Jeuna Díaz Manchay).

Recibido el 7 de septiembre de 2018; aceptado el 14 de enero de 2019
Objective: To describe the participation of the family caregiver for the comfort of people over 65 hospitalized, according to the perception of nurses and family caregivers in a public hospital in Lambayeque, Peru. Methods: Descriptive exploratory qualitative research, the sample was 20 informants with informed consent. The data was collected through the semi-structured interview and processed according to thematic content analysis. Results: Three categories emerged: a) Participation in basic care: Body comfort; $b$ ) Strategies for rest and sleep: Comfort of the environment; $c$ ) Affection, encouragement and faith: Contribution to emotional and spiritual comfort.

Conclusions: The hospital stay generates discomfort in older adults, but the family caregiver actively participates in the satisfaction of basic needs such as hygiene, food, laying of bed, avoidance of pain and noise, adequate lighting, safety, affection, hope and faith; care oriented to provide a peaceful and pleasant environment that helps them in rest and sleep, adaptation, recovery and quality of life. It is essential that the nursing staff trains the family caregiver and prepares them to collaborate in the care of both the hospitalization and the home.

KEYWORDS: Comfort, hospitalization, family caregiver, old person, nursing. 


\section{- INTRODUCCIÓN}

A nivel mundial, en 2020 hay más personas mayores de 60 ańos que niños menores de 5 años, y para el año 2050 , el $80 \%$ de las personas mayores vivirá en países de ingresos bajos y medios ${ }^{1}$. En el Perú, la proporción de población adulta mayor ha aumentado del $5,7 \%$ en el año 1950 al 10,4\% en 2018. El 42,2\% de los hogares del país tenía entre sus miembros al menos una persona de 60 o más ańos de edad. El 48,0\% de las personas mayores del país han padecido algún síntoma o malestar y el 37,5\%, alguna enfermedad o accidente ${ }^{2}$; aspectos que conllevan hospitalizaciones e implican necesidades de apoyo de la familia para sentirse confortado.

Una necesidad sentida en el adulto mayor reclama confort, el cual articula una experiencia inmediata y holística de vigorizarse cuando se afrontan sus necesidades; dicho afrontamiento abarca tres tipos de confort: alivio, tranquilidad y trascendencia, y engloba su contexto físico, psicoespiritual, ambiental y social ${ }^{3}$. De este modo, el confort se ha convertido en una necesidad que tiene la persona a lo largo de la vida, la salud y la enfermedad, y específicamente en grupos de pacientes socialmente vulnerables y fragilizados, como es el caso de las personas mayores que padecen enfermedades crónicas y buscan ayuda en las instituciones de salud. En situaciones de internamiento, experimentan cambios e insatisfacciones que conllevan sufrimiento e incomodidad; por ello, el enfermero/a debe tener la habilidad necesaria para proporcionar comodidad ${ }^{4}$. En el contexto hospitalario es indispensable la participación del familiar cuidador, el cual realiza cuidados genéricos (satisfacción de necesidades básicas), y en su mayoría entabla una relación genuina con la enfermera, de tal manera que la estancia hospitalaria sea acogedora y confortable.

Lo mencionado se evidencia en el estudio de Parihuaman ${ }^{6}$, quien establece que el familiar cuidador satisface las necesidades de confort en la hospitalización, y es responsable de la alimentación, el baño, acicalamiento matutino, cambio de pañal, movilización, masajes y administración de medicamentos orales, pero principalmente brindan afecto, lo que genera seguridad y tranquilidad. Villar et al. ${ }^{7}$ asienten que la cuidadora principal de ancianos hospitalizados es la mujer, la cual realiza actividades tales como acompańamiento, relación entre el anciano y los profesionales de la salud, además de apoyar en el aseo y la medicación, y enfatizan que si los familiares dejaran de hacer lo que hacen, sencillamente no sería posible la satisfacción de las necesidades biopsicosociales. Asimismo, el familiar cuidador consigue llevar objetos recreativos al hospital como televisor, radio y teléfonos móviles para que el paciente desvíe su preocupación hacia la distracción en comunicación con sus seres queridos; al mismo tiempo, recurre a la oración y lectura de la biblia mostrando fortaleza espiritual para colaborar en afrontar la enfermedad 8 , aspectos todos ellos que generan comodidad y confort a la persona mayor hospitalizada.

Objetivo del estudio: describir la participación del familiar cuidador para el confort de las personas mayores de 65 años hospitalizadas según la percepción de las enfermeras y los familiares cuidadores en un hospital público de Lambayeque, Perú.

\section{- MÉTODOS}

La presente investigación cualitativa fue de tipo exploratorio descriptivo\%. El muestreo se organizó por conveniencia y la muestra saturó con 10 familiares cuidadores de las personas mayores internadas y 10 enfermeras que trabajan en el servicio de medicina de un hospital público de mediana complejidad en Lambayeque, Perú, donde no existe el área geriátrica. Cada participante fue designado con un código: $F$ (familiar cuidador) y E (enfermera), acompañado del número de entrevista. Se excluyó del estudio a las enfermeras que estuvieron de vacaciones o habían participado en la prueba piloto, así como a familiares cuidadores que solo hablaban quechua, ya que las investigadoras no dominan ese idioma. De ahí la necesidad de hacer posteriores estudios en relación al confort y la cultura.

Las autoras declaran que son externas y no trabajan en esta institución de salud.

Para recolectar los datos, las investigadoras usaron la entrevista semiestructurada' de elaboración propia y validada por juicio de expertos. Una vez aprobada la investigación por el Comité de Ética de la Facultad de Medicina de la Universidad Católica Santo Toribio de Mogrovejo (Resolución N²46-2017-USAT-FMED) y obtenido el permiso de la institución para la ejecución, se identificaron los sujetos a estudio a quienes se les informó de su realización;: luego se solicitó la firma del consentimiento informado y, según su disponibilidad, se ejecutaron las entrevistas en la jefatura del servicio de medicina del hospital público, a través de un grabador de voz. La duración fue de 30 minutos. Generalmente, se realizaron en horario de noche por considerarse más tranquilo, sin bullicio, ni interrupciones.

Culminada la recolección de datos, se procedió a la transcripción, codificación y categorización según el análisis de contenido temático, y se hizo de forma manual. Este proceso se resume en los siguientes pasos ${ }^{10}$ :

A. Preanálisis, basado en la lectura y relectura de las entrevistas para identificar ideas principales relacionadas con el tema y los objetivos del estudio, tratando de contextualizarlo.

B. Proceso de codificación, mediante la identificación de palabras, frases o párrafos que tienen una significación.

C. Categorización, alcanzada por la agrupación por similitudes para obtener categorías, que significa darle una denominación que emerge de la segmentación de los discursos más relevantes y significativos desde el punto de vista del interés investigativo. Finalmente, se trianguló la información de los familiares cuidadores con la información dada por las enfermeras.

Durante el desarrollo de la investigación se consideraron tres principios de la ética personalista ${ }^{11}$. El principio de respeto a la vida humana, el principio de libertad y responsabilidad y el principio de socialización y subsidiaridad. Los sujetos del estudio no fueron expuestos a experimentación, ni sufrieron ningún daño físico ni emocional, participaron de manera voluntaria en el estudio, firmaron el consentimiento informado y su identidad fue protegida por códigos. Asimismo, se consideraron los criterios de rigor científico ${ }^{8}$ l la credibilidad, auditabilidad y transferibilidad. Posterior a la transcripción de las entrevistas recolectadas, los informantes las revisaron para dar su conformidad. Los datos obtenidos se guardarán durante 5 años tras la ejecución de la información (abril-junio del 2017), para la verificación de la información; los resultados podrían aplicarse en contextos parecidos.

\section{RESULTADOS}

La muestra en estudio tuvo las siguientes características: el 70\% lo conformó familiares por consanguinidad (hijas, hermanas, nietas) y el 30\% por afinidad (esposas); en su totalidad, mujeres entre 30 y 60 ańos. El $80 \%$ de las enfermeras hacía más de 10 ańos que trabajaban en este servicio y el 20\% solo 4 años; el $90 \%$ eran docentes en las diferentes universidades locales. A continuación, se relata la participación de familiares cuidadores para el confort de personas mayores hospitalizadas, clasificados en las siguientes categorías. 


\section{Participación en los cuidados básicos: confort del cuerpo}

El familiar cuidador participa en la satisfacción de necesidades básicas como la higiene, alimentación, eliminación, cambios de posición y alivio del dolor, como se evidencia en los discursos de las enfermeras y familiares cuidadores:

"El familiar da los alimentos a la persona mayor, pero cuando tiene sonda nasogástrica (SNG) le enseńamos cómo darle los alimentos y el cuidado que deben tener" (E1).

"Cuando le traen su comida yo lo siento y le doy la comida, pero primero veo que esté fría para que no se queme, le gusta la comida tibia" (F2).

"En las mañanas le brindamos una tina de agua para que el familiar le realice el aseo antes de que pase la visita médica, lo peinan y proceden al cambio de ropa para generar confort al paciente" (E6).

"El baño lo realizamos a diario, siempre en las mañanas; la llevo a la ducha, la siento en un banco y la comienzo a bañar" (F5).

"Le cambian el pañal con ayuda del técnico de enfermería [...] además educamos a los familiares para que movilicen al paciente por si no puede moverse solo, pues nosotras no nos alcanzamos para ello" (E9).

"Le cambio de pañal cuando se ensucia con la ayuda de la señorita de blanco (personal técnico) [...] muevo su cuerpo para que no le salgan escaras [...] si tuviese algún dolor o fiebre aviso a la enfermera" (F9).

"Nosotros le calmamos el dolor administrando los medicamentos que el médico indica; además les hacemos masajes o le indicamos al familiar cómo hacerlo" (E4).

"Cuando él tiene dolor llamo a la enfermera para que le ponga algo para el dolor y mientras llega le hago masajes en sus piernas y brazos para que se relaje" (F3).

La enfermera se apoya en el familiar cuidador para satisfacer las necesidades de la persona mayor hospitalizada, quienes están pendientes y vigilantes en brindar la comodidad mínima haciendo confortable la estancia hospitalaria.

\section{Estrategias para el descanso y sueño: confort del ambiente}

Por otro lado, el familiar cuidador realiza diferentes actividades de distracción y evitación de molestias en el entorno hospitalario favoreciendo el descanso y sueño de la persona mayor, lo cual se verifica en los siguientes discursos de enfermeras y familiares cuidadores:

"En la noche lo abrigan y evitan que haya ruidos y luz, pero por el tratamiento a veces los despertamos" (E8).

"Antes que duerma se lava su cara y los dientes, luego tiendo su cama y la abrigo, así puede estar tranquila" (F5).

"Las camas, los colchones están deteriorados y son incomodos, además no cuentan con barandas para la protección de mi papá. A veces no hay colchas ni sábanas" (F4).

"El familiar cuidador acomoda la cama y baja la cabecera, traen de casa colchas, almohadas, y una radio para su música favorita" (E5).

"Tomo su mano hasta que ella duerme... le pongo una canción para que se duerma" (F2)

"Apago las luces y pido que no haga bulla, porque eso le incomoda a mi papá y no puede dormir [...] los colchones se hunden, y los veladores están viejos" (F7).

Las estrategias para el descanso y sueño son velar por la comodidad en la cama, los familiares traen ropa de cama de su casa porque no hay en el hospital, escuchan música y los abrigan, evitan ruidos y luces para que la persona mayor duerma. Sin embargo, existen incomodidades como el deterioro de las camas, veladores y falta de barandas, que son reconocidas básicamente por los familiares.

\section{Afecto, ánimo y fe: contribución al confort emocional y espiritual}

Enfermeras y familiares cuidadores manifiestan que la persona mayor se siente tranquila en el hospital porque su familia lo acompańa, le da afecto, consuelo y favorece su fe:

"El familiar cuidador brinda tranquilidad y apoyo emocional a la persona mayor a través de un abrazo y diciéndole que lo quieren y lo esperan en la casa" (E2).

"Le digo que mis hijos lo extrañan y que ya quieren que pronto se recupere pues todos lo queremos mucho y queremos tenerlo en casa" (F9).

"Le dan fortaleza y le dicen que luche contra la enfermedad para que esté tranquilo y que no tenga preocupaciones" (E7).

"Le digo que se ve mejor y que cada día está mejorando, también le comento que pronto nos iremos al campo a disfrutar del paisaje a comer fruta y verdura fresca, su mazamorra, ver sus pollos" (F3).

"Leen la biblia, ponen estampitas y rosarios en los veladores de los pacientes para que siempre cuiden de ellos y les haga el milagro de sanarlos de sus enfermedades" (E8).

"Le gusta que le lean la biblia, que le ponga su estampa de la virgen María, digo que Dios no lo va a dejar solo, que él lo está cuidando, que no pierda la fe y que pronto se recuperará e iremos a la iglesia juntos como antes" (F10).

"No somos católicos, pero dejan entrar al pastor con un grupo de oración y mi mamá se sienta más segura con ellos y así ella está más tranquila" (F5).

Los familiares cuidadores dan afecto y ánimos para regresar a casa, intentan mantener la conexión con el hogar, además respetan y fomentan sus creencias religiosas, usando la lectura de la biblia, oración, y estampitas de santos, aspectos que le generan confort emocional y espiritual.

\section{- DISCUSIÓN}

El confort o comodidad es una necesidad básica del ser humano y un resultado esencial para el cuidado de la salud ${ }^{3,12}$. En esta investigación se describe la participación de familiares cuidadores para el confort de personas mayores hospitalizadas en su contexto físico-corporal, ambiental y psicoespiritual.

Actualmente, asistimos a un crecimiento exponencial del grupo de población de personas mayores. Como consecuencia, dicha situación presenta desafíos para el personal de salud que trabaja en hospitales con alta demanda de personas en esta etapa de la vida con varias de sus necesidades alteradas.

En ocasiones, para el personal de salud resulta difícil dedicar el tiempo necesario para desarrollar actividades que contribuyan a la comodidad, como acompañarle a comer o ir al baño, llamar a parientes, etc. Por ello, se recurre al apoyo de otros como sucede en un departamento de emergencias, el cual ha implementado el apoyo de un equipo de voluntarios dirigido por la organización benéfica Age UK para la ayuda a las personas mayores en algunas de estas actividades ${ }^{13}$. Contrariamente, en los países latinoamericanos como en el Perú, cuando una persona mayor es hospitalizada, lo acompaña permanentemente un familiar cuidador, y 
colabora en los cuidados básicos como la higiene, lubricación de la piel, cambio de posición, masajes y alimentación, entre otros ${ }^{14}$.

En este estudio, por la condición de dependencia de las personas mayores, hay un declive de sus capacidades funcionales y de su autonomía para realizar las actividades de la vida diaria, tan básicas y necesarias como alimentarse, bańarse, asearse e ir al baño, por lo que requieren de forma permanente la ayuda de un familiar cuidador de sexo femenino, según las normas institucionales del escenario de la investigación. Los hallazgos del estudio encuentran semejanzas con los estudios de Flores et al. $^{15}$, ya que el familiar cuidador, no solo colabora en el baño del adulto mayor, sino que lo ajusta a sus necesidades, sin dejar de lado sus costumbres y creencias, como el horario del baño, y los materiales que utiliza; muchos de estos materiales son traídos de su hogar. De esta manera, realizan la higiene matutina, bańo de esponja o acompańan a la ducha para evitar caídas; también cambian de vestimenta, de pañales y los peinan, para que estén presentables y se sientan cómodos. Es más, el estudio de Escobar et al. ${ }^{16}$ establece que el familiar cuidador no solo asume el cuidado básico, sino también el de mayor complejidad, como la supervisión de goteros, el cuidado de la sonda vesical y de los catéteres, además de identificar cambios y posibles complicaciones, las cuales son informadas al personal de enfermería.

El estudio refleja que los familiares dan de comer a la persona mayor. Si el paciente tiene SNG, la enfermera enseña y explica la forma correcta de alimentarlo; asimismo, incide en que no ingieran alimentos que pueden alterar su estado de salud. Pero, el ambiente hospitalario influye directamente en la reducción del apetito, especialmente cuando hay presencia de olores desagradables que causan malestares gástricos innecesarios a los pacientes ${ }^{17}$.

Cabe resaltar que una de las mayores incomodidades que tiene el adulto mayor es el dolor, incomodidad apremiante de cuidado, ya que constituye una experiencia sensorial y emocional desagradable. Por tanto, es necesario utilizar un tratamiento eficaz para controlar el dolor, lo que mejora la calidad asistencial y proporciona mayor confort ${ }^{18}$. Por ello, los familiares del estudio junto con la enfermera cumplen un papel fundamental para mitigar el dolor; el familiar realiza cambios de posición para evitar las ulceras de presión; también hace masajes, estimulando la producción de endorfinas que ayudan a reducir o aliviar el dolor, generando mayor bienestar.

Por lo expuesto, es significativo establecer las funciones propias del personal de enfermería, así como del familiar cuidador, y sobre todo el cómo se capacita al familiar sobre procedimientos más específicos. Es ahí donde el personal de salud debe ampliar y fundamentar un plan de educación para la salud del familiar hospitalizado en el que se consideren tanto los cuidados básicos como los específicos para poder corroborar que se realizan y vigilan de manera adecuada, ya que de lo contrario se estaría afectando a la salud del paciente si no se verifican bien esos cuidados que propiamente son parte fundamental del personal de enfermería, porque no se trata de dar toda la responsabilidad del paciente al familiar cuidador y que el personal de enfermería solo sea su supervisor, sino de ser un equipo para el confort de nuestro paciente.

Asimismo, un ambiente o entorno saludable genera en el paciente confort y bienestar durante su estancia hospitalaria, mediante un ambiente limpio, iluminado, ventilado y evitando ruidos incómodos que impiden el adecuado descanso y sueño durante su estancia ${ }^{6}$. Cabe mencionar que con el envejecimiento se reduce la intensidad, duración y continuidad del sueño; además, existen factores como el frío, la luz, el ruido, el consumo de cafeína y la deambulación frecuente del personal de enfermería en la noche, que en cierto modo intervienen en la alteración del sueño en la persona mayor y afectan a su estado de salud general y anímico ${ }^{19}$. También, la comodidad o confort para lograr el descanso y el sueño de la persona mayor se obtiene con una cama bien arreglada donde su cuerpo encuentra bienestar, sumado al uso de distractores que consiguen aliviarlo de tensiones y conciliar el sueño. La acción de abrigar permite que la temperatura corporal de la persona mayor se conserve, y colocar ropa de cama limpia libre de malos olores mejora la comodidad y adaptación al ambiente extraño del hospital ${ }^{20}$.

Sin embargo, en esta investigación se evidenció que las personas adultas mayores son muy sensibles al ruido causado por el sonido de la cama, las luces encendidas y por el bullicio de algunos familiares, creando molestias que interrumpen su descanso y sueńo. Por esta razón, un aspecto importante que influye en la comodidad del paciente durante su hospitalización es la eliminación de ruidos. Se encuentra pendiente, por otra parte, la necesidad de mejorar las camas, las barandas, los veladores y las cortinas debido a la falta de recursos; el uso y la antigüedad de estos muebles hace que estén ya deteriorados, y generan un riesgo para la salud de la persona mayor, ya que afecta a su comodidad y confort.

Igualmente, el familiar cuidador cumple un rol importante a la hora de brindar apoyo emocional a su paciente, ya que le expresan ternura a través de un abrazo, un gesto de carińo, decir un te quiero, que nunca los van a dejar solos..., permanecen a su lado con presencia y acompañamiento, infundiéndoles tranquilidad para afrontar la enfermedad, les dan fuerzas para seguir adelante y encontrar sentido a la misma ${ }^{6}$. Por otra parte, se observó en este estudio que la intervención musical y el tacto son formas de comunicación no verbal que constituyen intervenciones de atención. Dichas acciones son esenciales para el establecimiento de la comunicación y el intercambio de ideas y emociones, ya que transmiten la comodidad, el afecto, el bienestar, la confianza, la seguridad y el intercambió ${ }^{4}$.

Así, el confort espiritual en las personas mayores se da a través de la fe religiosa al rezar o leer la biblia, lo cual les permite nutrir la esperanza, resignificar la experiencia y buscar un sentido a la vida, y adaptarse a convivir con la enfermedad ${ }^{21}$. La espiritualidad como componente de la corporalidad junto al de la salud siempre está presente para los seres humanos que se encuentran en situación de enfermedad, ya sea crónica o terminal, y para los que se encuentran cerca del enfermo; el bienestar espiritual es un factor de crecimiento personal, un aspecto de trascendencia que los acerca a muchas respuestas necesarias para dar sentido a su vida, a la cotidianeidad, el dolor y el sufrimiento humano ${ }^{22}$. Cabe afirmar que durante la investigación se evidenció que en los veladores de los pacientes había estampitas de imágenes religiosas, crucifijos y biblias a los cuales recurren los pacientes para sentir la presencia de Dios y sentirse acompañados en todo momento. Ratifica la investigación de $\mathrm{Pa}$ rihuaman ${ }^{6}$ al encontrar en su estudio cómo los pacientes recurren a Dios usando las estampitas, imágenes religiosas, crucifijos y biblias presentes en sus veladores, así como los rezos y pláticas religiosas de los familiares y enfermeras que los atienden, para reforzar el confort de los pacientes y así mejorar su estado de salud. Resulta fundamental que se respeten las costumbres y creencias religiosas de la persona mayor.

\section{- CONCLUSIONES}

El rol del familiar cuidador en el confort de la persona mayor hospitalizada es fundamental, y se complementa con la misión de la enfermera en las actividades de cuidado para la satisfacción de las necesidades básicas: la limpieza e higiene, alimentación, tendido de cama, movilización, masajes, iluminación adecuada y evitando el ruido; todas orientadas a proporcionar un entorno tranquilo y agradable para el descanso y sueńo en la recuperación del paciente. Así mismo, la presencia del familiar en la hospitalización les proporciona tranquilidad, seguridad, afecto, esperan- 
za, fe y respeto por sus creencias religiosas. De este modo contribuyen a brindar un confort corporal, ambiental, emocional y espiritual, a pesar de las deficiencias que existen en el hospital.

El cuidado profesional de enfermería se debe manifestar en el cuidado de las necesidades básicas del paciente tanto en el hospital como en casa, apoyándose en el familiar cuidador durante el proceso de recuperación y cuidado de este, por lo que se debe implementar una adecuada información para garantizar el manejo en la calidad de la atención del paciente para obtener un mayor confort en sus cuidados.
Con el reconocimiento de las múltiples necesidades básicas de las personas mayores en el hospital, es un hecho de gran relevancia para los profesionales de enfermería conocer a fondo la experiencia vivida de los cuidadores de personas mayores hospitalizadas, con el propósito de elaborar programas específicos para la capacitación adecuada de los cuidadores primarios de estos pacientes que colaboran en el bienestar y confort tanto en la hospitalización como en el hogar, para garantizar la calidad de atención

\section{Conflicto de intereses}

Las autoras declaran no tener ningún conflicto de intereses.

\section{BIBLIOGRAFÍA}

1. Organización Mundial de la Salud. Informe Mundial sobre el envejecimiento y la salud [Internet]. Estados Unidos de América: Organización Mundial de la Salud; 2015 [acceso 6 de septiembre de 2017]. Disponible en: http://apps.who.int/iris/bitstre am/10665/186466/1/9789240694873_spa.pdf

2. Instituto Nacional de Estadística e Informática del Perú (INEI). Situación de la Población Adulta Mayor. Enero-Febrero-Marzo 2018 [Internet]. Lima: INEl; Junio 2018. Informe Técnico № 2 [acceso 3 de agosto de 2018]. Disponible en: https://www.inei.gob.pe/media/ MenuRecursivo/boletines/01-informe-tecnico-n02 adulto enefeb mar2018.pdf

3. Kolcaba K, Tilton C, Drouin C. Comfort Theory: a unifying framework to enhance the practice environment. JONA [Internet]. 2006 [acceso 13 de mayo de 2017];36 (11):538-44. Dispobible en: https://journals. Iww.com/jonajournal/Abstract/2006/11000/Comfort_Theory_A Unifying_Framework_to_Enhance.10.aspx

4. Pontifice V, Cruz Dourado R, Pontifice R. Geriatric care: ways and means of providing comfort. Rev. Bras. Enferm [internet]. Julio-agosto 2017 [acceso 13 de noviembre de 2017]; 70(4): 865-72 Disponible en: http://dx.doi.org/10.1590/0034-7167-2016-0636

5. Arica V, Diaz R. El cuidado de enfermería al adulto mayor hospitalizado: complementariedad, calor humano e interculturalidad. Acc Cietna [internet]. 2017 [acceso 13 de julio de 2018];4(1):35-47. Disponible en: http://revistas.usat.edu.pe/index.php/cietna/article/ view/30

6. Parihuaman Y. El confort en el cuidado de enfermería a la persona oncológica según la teórica Katharine Kolcaba, 2016 [tesis de licenciatura]. Chiclayo: Universidad Católica Santo Toribio de Mogrovejo: 2016.

7. Villar R, Pancorbo P, Jiménez M, Cruz A, García P. Qué hace el cuidador familiar en el hospital. Cómo se ve a sí mismo y cómo lo ven los profesionales. Gerokomos [Internet]. 2012 [acceso 13 de noviembre de 2017];23(1):7-14. Disponible en: http://scielo.isciii.es/pdf/ge roko/v23n1/comunicacion1.pdf

8. Díaz R, Bustamante S. Enfermería, familia y persona con cánce terminal del cuidado hospitalario al cuidado en el hogar. Trujillo: Editora Gráfica Emmanuel; 2011

9. Grove S, Gray J, Burns N. Investigación en enfermería. Desarrollo de la práctica enfermera basada en la evidencia. 6. ${ }^{a}$ ed. Barcelona: Elsevier; 2016.

10. Do Prado M, De Souza M, Monticelli M, Cometto M, Gómez P, eds Investigación cualitativa en enfermería. Metodología y didáctica. Washington DC: Organización Panamericana de la Salud; 2013

11. Sgreccia E. Manual de Bioética. Fundamentos y ética biomédica. Madrid: Talisio; 2015.

12. Pinto S, Caldeira S, Martins JC, Rodgers B. Evolutionary Analysis of the Concept of Comfort. Holist Nurs Pract [Internet]. Julio/Agosto 2017 [acceso 13 de mayo de 2017];31(4):243-52. Disponible en https://www.ncbi.nlm.nih.gov/pubmed/28609409

13. Triggle N. Providing comfort and support to older people. Emerg Nurse [Internet]. Octubre 2012 [acceso 13 de noviembre de 2017];20(6):10-3. Disponible en: https://www.ncbi.nlm.nih.gov/ pubmed/23167006

14. Martín E. El cuidado familiar. Una revisión sobre el perfil y la realidad de las personas cuidadoras en el hospital. Rev Paraninfo Digital [Internet]. 2017 [acceso 11 de noviembre de 2017];XI(27). Disponible en: http://www.index-f.com/para/n27/pdf/115.pdf

15. Flores E, Rivas E, Seguel F. Nivel de sobrecarga en el desempeño del rol del cuidador familiar de adulto mayor con dependencia severa. Ciencia y Enfermería [internet]. Abril 2012 [acceso 27 de julio de 2018];XVIII (1). Disponible en: http://www.redalyc.org/articulo. oa? id=370441809004

16. Escobar CL, Cardozo Y, Mejía OL, Muñoz Cardona MJ, Cuartas PA Significado que construye el personal de enfermería sobre los cui- dadores familiares de personas hospitalizadas. Investigación en Enfermería: Imagen y Desarrollo [Internet]. Enero-junio 2016 [acceso 11 de julio de 2018];18(1):79-93. Disponible en: http://www.redalyc. org/articulo.oa?id=145243501006

17. Lima D, Pelzer M, Barros Edaiane J, Semedo Deisa S, Rosales R. Factores que dificultan la alimentación por vía oral del anciano hospitalizado. Enferm. glob [Internet]. 2017 [acceso 27 de julio de 2018];16(48):429-64. Disponible en: http://scielo.isciii.es/scielo. php?script=sci_arttext\&pid=\$1695-61412017000400429\&lng=es

18. Castel-Bernal B, Garcia-Vardón V, Tornero-Molina J. Evaluación psicológica en el dolor crónico. Reumatol Clin [Internet]. Nov 2017 [acceso 9 de diciembre de 2017];2(Supl 1):44-9. Disponible en: https:// www.reumatologiaclinica.org/es-pdf-S1699258X06730820

19. Jurado 0 , Pinta A Vallejo Y Villareal M. Trastornos del sueño en adultos mayores de la ciudad de Pasto. Criterios [Internet]. 2016 [acceso 9 de agosto de 2017];23(1):369-82. Disponible en: http:// editorial.umariana.edu.co/revistas/index.php/Criterios/article/ view/1803/1866

20. Aguilera L, Diaz M, Sánchez H. Trastornos del sueño en el paciente adulto hospitalizado. Rev Hosp Clín Univ Chile [Internet]. 2014 [acceso 9 de agosto de 2017]:23:13-22. Disponible en https://www. redclinica.cl/Portals/0/Users/014/14/14/Publicaciones/Revista/ trastorno_sueno_hospitalizado.pdf

21. Gil K, Díaz R, Cervera M. La Corporalidad en el Cuidado de Enfermería a la Persona con cáncer en Fase Terminal. Complementariedad: Enfermera-Familiar acompañante. Chiclayo: Universidad Católica Santo Toribio de Mogrovejo; 2015

22. Ortega M, Ojeda MA, Ortiz A0, Guerreo R. Espiritualidad y cuidado de enfermería. Revista Iberoamericana de las Ciencias Sociales y Humanísticas [Internet]. Junio-Diciembre 2016 [aceso 1 de agosto de 2018];5(10). Disponible en: https://dialnet.unirioja.es/descarga/ articulo/5662592.pdf 